Der Leser möge selbst das Studium der entsprechenden Gleichung (17) vornehmen und sich namentlich mit dem Temperaturzustand in den Ebenen $\psi_{0}-\varepsilon_{0}=0, \pi / 2, \pi$ näher befassen.

\title{
Summary
}

New particular solutions are given for certain types of nonhomogeneous Bessel equations. Previous considerations allow of considerable extension and their technical importance is illustrated by an example in heat conduction.

(Eingegangen: 4. April 1959.)

Varia - Miscellaneous - Divers

\section{6th National Symposium on Reliability and Quality Control in Electronics, Statler Hilton Hotel, Washington, D. C., USA.}

In Washington findet vom 11. bis 13. Januar 1960 das 6. National Symposium on Reliability and Quality Control statt. Der $Z_{\text {weck }}$ dieser Tagung, welche vom Institute of Radio Engineers (IRE) und anderen wissenschaftlichen Vereinigungen durchgeführt wird, ist, Spezialisten, welche auf dem Gebiet der Betriebssicherheit und Qualitätsüberwachung über Erfahrung verfügen, zusammenzuführen. In Vorträgen und Diskussionen wird ein ausgiebiger Gedankenaustausch gepflegt. Programme und Anmeldeformulare sind $z$ u beziehen bei C. M. BEYER, OASD, Room 3D1031. The Pentagon, Washington 25, D.C., USA.

Buchbesprechungen - Book Reviews - Notices bibliographiques

Elementare Informationstheorie. Von HEINZ ZEMANEK (R. Oldenbourg, Wien und München 1959). 120 S., 28. Abb., 2 Tabellen.

Der Verfasser versucht im vorliegenden Büchlein die Informationstheorie in einer dem Ingenieur zugänglicheren Form darzustellen. Er unterscheidet zunächst 2 Hauptprinzipien, welche getrennt betrachtet werden können. Zunächst wird das logische Netz entwickelt, das heisst die Form, in welcher sich "Information" ausdrücken lässt. Dazu gehören die verschiedenen Kodierungsmöglichkeiten. Eine umfangreichere Betrachtung ist der Information als statistischer Vorgang gewidmet. Die Entwicklung des optimalen realisierbaren Kodes, zum Beispiel mit BinärZeichen, wird auf geometrischem Wege in Abweichung zu Shannon entwickelt, wobei die angegebene Methode eine kleinere Redundanz ergibt. Beispiele über Informationsgehalt werden aus der Sprache und der Biologie besprochen. Ein drittes Kapitel ist der statistischen Zuordnung gewidmet, welche eine Rolle spielt bei der gestörten Übermittlung, das heisst bei der Berechnung der maximalen Kanalkapazität eines Übertragungsvorganges bei Anwesenheit von Störungen. Der Übergang 